\title{
Neutrophil-to-lymphocyte ratio for predictor of in-hospital mortality in ST-segment elevation myocardial infarction: a meta-analysis
}

\author{
Rodry Mikhael, ${ }^{1}$ Evan Hindoro, ${ }^{2}$ Sharleen Taner, ${ }^{3}$ Antonia Anna Lukito ${ }^{4}$
}

Check for updates

pISSN: 0853-1773 • elSSN: 2252-8083 https://doi.org/10.13181/mji.oa.202795 Med J Indones. 2020;29:172-82

Received: August 12, 2018 Accepted: February 19,2020

Authors' affiliations:

${ }^{1}$ Faculty of Medicine, Universitas Indonesia, Jakarta, Indonesia, ${ }^{2}$ Bengkalis General Hospital, Riau, Indonesia, ${ }^{3}$ Faculty of Medicine, Universitas Pelita Harapan, Tangerang, Indonesia, ${ }^{4}$ Department of Cardiology, Universitas Pelita Harapan, Tangerang, Indonesia

\section{Corresponding author:}

Evan Hindoro

Bengkalis General Hospital, Jalan Kelapapati Tengah, Bengkalis, Riau 28711, Indonesia

Tel/Fax: +62-766-7008400

E-mail: evanhindoro@gmail.com

\begin{abstract}
BACKGROUND ST-segment elevation myocardial infarction (STEMI) is the most lifethreatening condition of acute coronary syndrome that carries a poor prognosis of inhospital mortality. Multiple scoring systems have been developed to predict in-hospital mortality and other cardiovascular events. Neutrophil-to-lymphocyte ratio (NLR) is hardly used as a predictor of in-hospital mortality. This study was aimed to determine the predictive value of NLR concerning in-hospital mortality in STEMI patients.
\end{abstract}

METHODS Literature search and pooled analysis related to studies on MEDLINE/ PubMed, EBSCO, Science Direct, Cochrane, and ProQuest were retrieved. Inclusion criteria were met if they were cohort studies, the subjects were STEMI patient, contained pretreatment NLR cut-off, and considered in-hospital mortality, which is defined as cardiac or all-cause mortality. Quality assessment was conducted using Newcastle-Ottawa scale. Review Manager version 5.3 (The Nordic Cochrane Centre, Copenhagen) was used for meta-analysis.

RESULTS We found 12 studies with a total of 7,251 STEMI subjects with median NLR cutoff value of 5.6. Elevated NLR on admission carries a high risk of in-hospital mortality (odds ratio $[\mathrm{OR}]=3.00,95 \%$ confidence interval $[\mathrm{Cl}]=2.46-3.67$ ). A slightly higher risk of all-cause mortality $(\mathrm{OR}=2.74,95 \% \mathrm{Cl}=1.99-3.77)$ was observed compared with cardiac-related mortality $(\mathrm{OR}=3.20,95 \% \mathrm{Cl}=2.47-4.14)$. No significant heterogeneity was observed between these studies $\left(p=0.46, I^{2}=0 \%\right)$.

CONCLUSIONS Elevated NLR predicts a higher in-hospital mortality rate of STEMI patients.

KEYWORDS in-hospital mortality, neutrophil-to-lymphocyte ratio, STEMI
Coronary heart disease ( $\mathrm{CHD}$ ) is known as the leading cause of death globally. ${ }^{1}$ Notably, the increase of morbidity rate has also increased a concern on mortality. Global and regional data have shown that males have a higher risk of CHD than fertile females. ${ }^{2}$ Based on findings from the National Cardiovascular Center Harapan Kita in 2014, the ratio of CHD in males versus females is $2.15: 1$, with mean age of 14-95 years old. ${ }^{3}$ One of the most daunting events of CHD is an acute coronary syndrome, a life-threatening condition that transpires due to a ruptured or eroded atherosclerotic plaque that is magnified by a thrombosis. Transmural myocardial infarction due to a total occlusion of atherothrombotic process in coronary blood vessels are shown in electrocardiography (ECG) results of persistent ST-segment elevation myocardial infarction (STEMI). ${ }^{4}$ Various immune-mediated inflammatory events occur after the rupture of atheroma.5,6 
Numerous scoring systems were established to predict major adverse cardiac events (MACEs), especially mortality in STEMI, which include thrombolysis in myocardial infarction (TIMI) score and the Global Registry of Acute Coronary Event score. ${ }^{4}$ However, hematologic score indices were developed to predict the mortality rate. Hematological indices are basic laboratory examinations that are widely available and can be used as a marker of any inflammation, including myocardial infarction. Among different hematological indices, neutrophil-to-lymphocyte ratio (NLR) has the highest predictive value in predicting an all-cause of death or myocardial infarction. ${ }^{7}$ NLR is known as a systemic inflammatory marker in numerous acute inflammatory cases. It is also recognized as the simplest, easiest, and most affordable marker to predict MACEs, especially in STEMI. ${ }^{8}$

Despite abundant published evidence, there is lack of meta-analysis that systematically evaluate, assess, and integrate, and bring the results to a solid conclusion. Thus, assessing the data and emphasizing the value of NLR in STEMI patients is worthwhile to determine the predictive value of NLR for MACEs, particular in-hospital mortality. This meta-analysis was aimed to investigate the value of NLR in STEMI as a predictor of in-hospital mortality.

\section{METHODS}

\section{Search strategy}

This meta-analysis was based on observational studies in epidemiology and recommendation of preferred reporting items for systematic reviews and meta-analyses (PRISMA) statement. This focused on studies that evaluate NLR as a predictive factor of inhospital mortality in STEMI. We included studies in which patients were diagnosed as STEMI. The primary endpoints were in-hospital mortality, which is defined as all-cause and cardiac-related mortality.

A systematic and comprehensive literature search was conducted using various search engines/ libraries for relevant publications, including PubMed/ MEDLINE, Cochrane, Science Direct, Proquest, and EBSCO databases from their inception to July 1, 2017. The following search terms were used: "myocardial infarction" or "STEMI", and "NLR." A reference list of the obtained abstract titles was screened by manual hand search to obtain additional articles. No language restriction was applied. We contacted some authors whose full text article were unavailable to obtain the required information. Additionally, we conducted a manual search by reviewing the citations in the included publications and reviewed the related references.

\section{Eligibility criteria}

Search results from the five aforementioned search engines/libraries were imported into Mendeley Reference Manager 1.17.11 (Elsevier, Netherlands) for duplicate deletion. Two reviewers (RM and $\mathrm{EH}$ ) independently screened the references using the predetermined eligibility criteria. The inclusion criteria were: (1) cohort studies, either prospective or retrospective design; (2) patients diagnosed with STEMI confirmed by elevated cardiac biomarkers and persistent ST elevation in two consecutive leads on ECG; (3) contained an evaluation of pretreatment NLR with a cut-off value for predicting mortality; (4) contained outcome of mortality (all-cause or cardiac-related); and (5) available as full text. We contacted the author if there is any doubt related to the studies, or if the studies were not available as full text. Exclusion criteria were as follows: (1) animal studies, review articles, letters, editorials, communication, and case reports with no original data; (2) studies that did not directly report odds ratio (OR) in which we could not reconstruct them and obtain the exact patient number; (3) studies published as a poster presentation or unavailability of full text after comprehensive searching or contacting the author; and (4) studies with insufficient data for estimation.

Different decisions or disagreements in the screening procedures were discussed between two reviewers to reach a consensus. Consultation from our supervisor (AAL) was conducted if necessary. The full texts of included references were then retrieved through the library of the Universitas Indonesia, and full text screening was subsequently conducted to identify relevant references for data extraction.

\section{Outcome of interest and definitions}

All outcomes were measured to analyze the predictive value of elevated NLR in STEMI patients to predict in-hospital mortality. The outcomes consisted of the following: (1) all-cause mortality, defined as any cause of deaths that happened during hospitalizations (deaths occurring after patients were transferred to another facility were not considered as in-hospital deaths); (2) cardiac-related mortality, defined as any 
death caused by cardiac disorders, such as sudden cardiac deaths, acute heart failure, cardiogenic shock, resuscitated cardiac arrest, and reinfarction.

\section{Data extraction}

After eligibility assessment, the two investigators (RM and $\mathrm{EH}$ ) independently extracted the following data according to a pre-specified protocol into a template that consisted of first author's name, year of publication, country of origin, number of patients, characteristic of patients (mean age and number of male patients/gender), study design, NLR cut-off, NLR determination method, duration of follow-up, therapy modalities, and type of outcomes measured.

\section{Quality assessment}

The quality of each selected study was assessed using the Newcastle-Ottawa scale, which included three criteria: selection, comparability, and outcome. Included studies were independently assessed by two reviewers (RM and EH). Studies achieving six or more stars were considered to be of good quality. Disagreements and discrepancies regarding the inclusion process were reconciled via consensus. Publication bias was evaluated through the visual analysis of the funnel plot asymmetry.

\section{Statistical analysis}

Meta-analysis was conducted according to recommendations from the Cochrane Collaboration (Oxford, United Kingdom). The effect outcomes were estimated by OR for dichotomous data and reported with $95 \%$ confidence intervals (Cls). Analysis was conducted with Review Manager 5.3 software (The Nordic Cochrane Centre, Copenhagen), and then we assessed and quantified the statistical heterogeneity for each pooled estimate using the Cochran's Q chisquare test and $I^{2}$ statistic. A chi-square value of $<0.05$ was considered statistically significant. Random effect model analysis was used when items had high levels of heterogeneity (with $I^{2}>75 \%$ ). In random effects model, we assumed that a variation between studies and thus the calculated OR has a more conservative value. Publication bias was assessed by funnel plot, while an asymmetry distribution in funnel plot increases the suspicion of publication bias. To avoid publication bias, reviewers have been searching in several databases for registered studies such as ClinicalTrials.gov, MEDLINE, and Cochrane.

\section{RESULTS}

Our initial search yielded 1,420 studies on the association of NLR and in-hospital mortality through an initial literature search. After final screening of titles and abstracts, 1,407 studies were excluded because they were either review articles, abstracts, duplicate reports, or studies irrelevant to the current analysis. After careful evaluation by applying our inclusion criteria, a total of 14 studies were identified. Two studies by Núñez et al ${ }^{9}$ and Sawant et al ${ }^{10}$ were excluded due to an insufficient data, although the original author had been contacted via email. Therefore, 12 studies with 7,251 patients, which met our inclusion criteria, were enrolled for this metaanalysis. ${ }^{11-22}$ The flowchart showed the process of study selection (Figure 1).

\section{Study characteristics}

The studies were published between 2010 and 2017, and the characteristics are summarized in Table 1. The studies were conducted in various countries. Four studies were conducted in Turkey, ${ }^{11,14,17,18}$ three in China, ${ }^{13,19,21}$ two in Korea, ${ }^{15,16}$ one each in Indonesia ${ }^{20}$ and in Pakistan, ${ }^{22}$ and one was performed in a multicenter setting ${ }^{12}$ (Table 1).

Primary percutaneous coronary intervention ( $\mathrm{PCl}$ ) as the reperfusion strategy was reported in eight studies. ${ }^{11-13,15-18,21}$ Two studies reported fibrinolysis as the main treatment, ${ }^{14,22}$ and the remaining studies reported mixed treatment of either primary $\mathrm{PCl}$ or fibrinolysis (with or without rescue PCI). 19,20 The NLR cut-off was determined using different methods in each study. Three studies determined the NLR cut-off from the receiving-operator characteristic curve analysis, ${ }^{11,16,20}$ whereas seven studies determined the NLR cut-off from tertiles (tertile 3 versus tertile $1 \& 2$ ), $12,14,15,17-19,21$ one study from the median of interquartile, ${ }^{13}$ and one study used the NLR cut-off from previous study. ${ }^{22}$

The sample size ranged from 165 to 2,410 . Mean or median age ranged from 55.37 to 62.9 years old. Percentage of male patients ranged from $72.3 \%$ to $86.3 \%$. Normality test of various NLR cut-offs showed a median value of 5.6 , with a minimum value of 4.4 and a maximum of 7.5 .

\section{Risk of bias}

The quality assessment on the Newcastle-Ottawa scale in each study was revealed (Table 2). All studies 


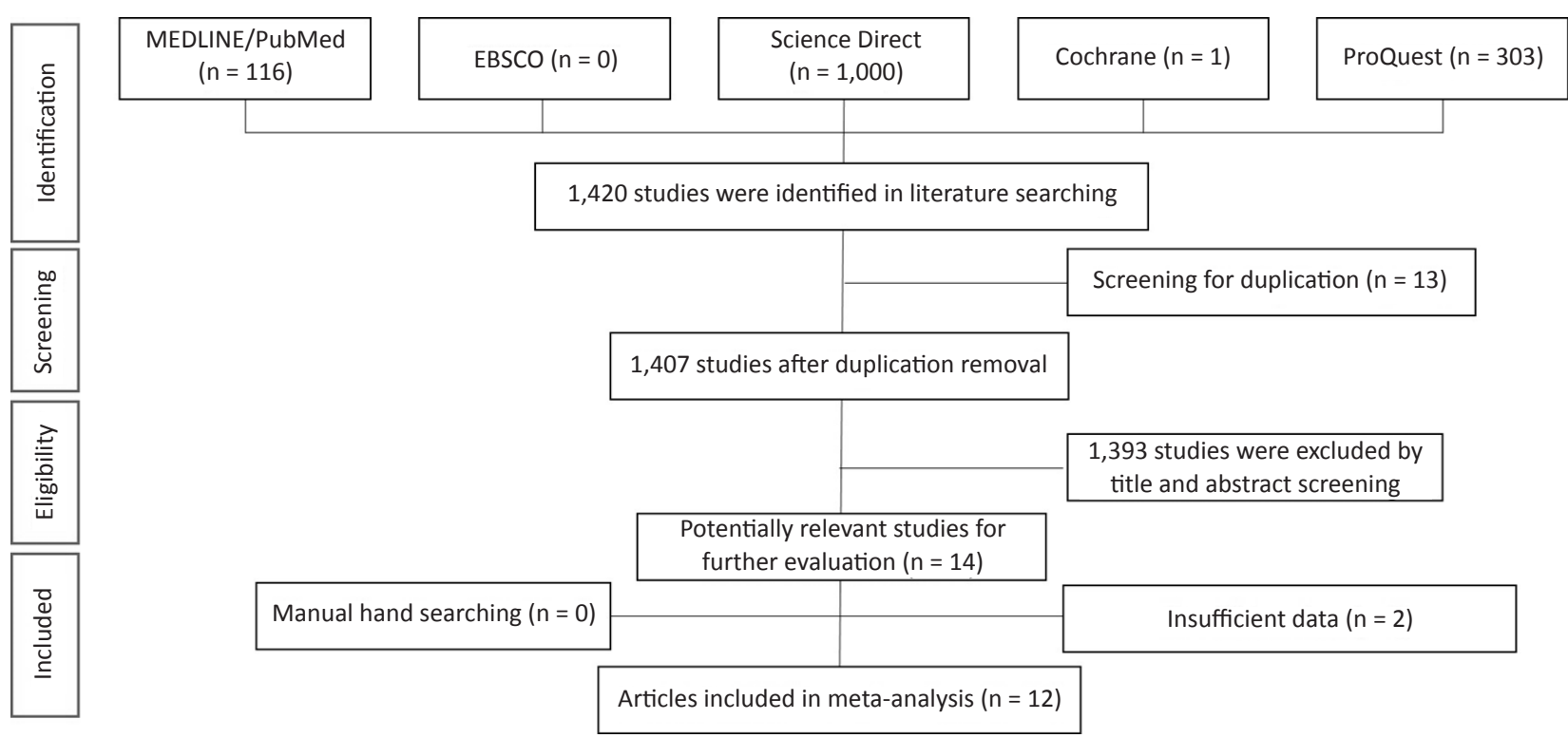

Figure 1. Flow chart of the study selection process. Twelve studies were found to be relevant with the inclusion criteria. Two studies were excluded due to insufficient data

were rated as having a good quality based on the selection and outcome criteria. For the comparability criteria, none of the OR of these studies were adjusted for the confounders listed, e.g., age, gender, infarct location, reperfusion strategies, and others. However, if each study described its baseline characteristics of the subjects with no significant differences, then the groups (low versus high NLR) can be considered comparable. All studies had a good quality, as the scores of the Newcastle-Ottawa quality assessment scales were higher than 6 .

All current evidence listed above showed that elevated NLR increased the risk of in-hospital mortality. This review had included all studies currently available in the field for the association of elevated NLR and inhospital mortality in STEMI patients. To minimize bias in the review process, two of the review authors selected studies, extracted the study data, and assessed the risk of bias and quality of the evidence independently.

As shown in Figure 2, the funnel plot appeared to be asymmetrical in the distribution of studies around the vertical axis. This condition suggests the possibility of publication bias or the "small-study effect" that is included in this study. However, chi-square test for objective heterogeneity analysis shows $p=0.46$ with $l^{2}$ $=17 \%$ (no significant heterogeneity).

\section{Forest plot}

The relationship of NLR cut-off with in-hospital mortality is described in Figure 3. A total of 275 events out of 2,860 patients with high NLR were observed, compared with 169 events in 5,083 patients with low $\operatorname{NLR}(\mathrm{OR}=3.00,95 \% \mathrm{Cl}=2.46-3.67)$.

A subgroup analysis was performed to see the performance of NLR in all-cause mortality or cardiacrelated mortality. Pooled analysis showed that high NLR increases the risk of both all-cause mortality (OR $=2.74,95 \% \mathrm{Cl}=1.99-3.77)$ and cardiac-related mortality ( $\mathrm{OR}=3.20,95 \% \mathrm{Cl}=2.47-4.14)$.

All results showed no substantial heterogeneity between each study based on the eye-ball test. Chisquare test was performed for objective heterogeneity analysis with $p=0.46$ and $l^{2}=0 \%(<75 \%)$. $X^{2}$ test value was less than degrees of freedom $(\mathrm{df})$ value $\left(X^{2}=0.56\right.$, $\mathrm{df}=1)$. These findings mean that our null hypothesis was rejected. Thus, no substantial heterogeneity between each study was found.

\section{DISCUSSION}

Among all studies that being analyzed, most subjects were male, $>50$ years old, and treated with various reperfusion strategies such as primary $\mathrm{PCl}$ and fibrinolytic. Almost all studies exclude malignancy, infectious disease, and chronic inflammatory condition to reduce cause-effect bias. This study can depict a real clinical situation, as most STEMI patients in many registries have a similar sociodemographic and clinical characteristic with subjects being analyzed in this study. ${ }^{23-25}$ 


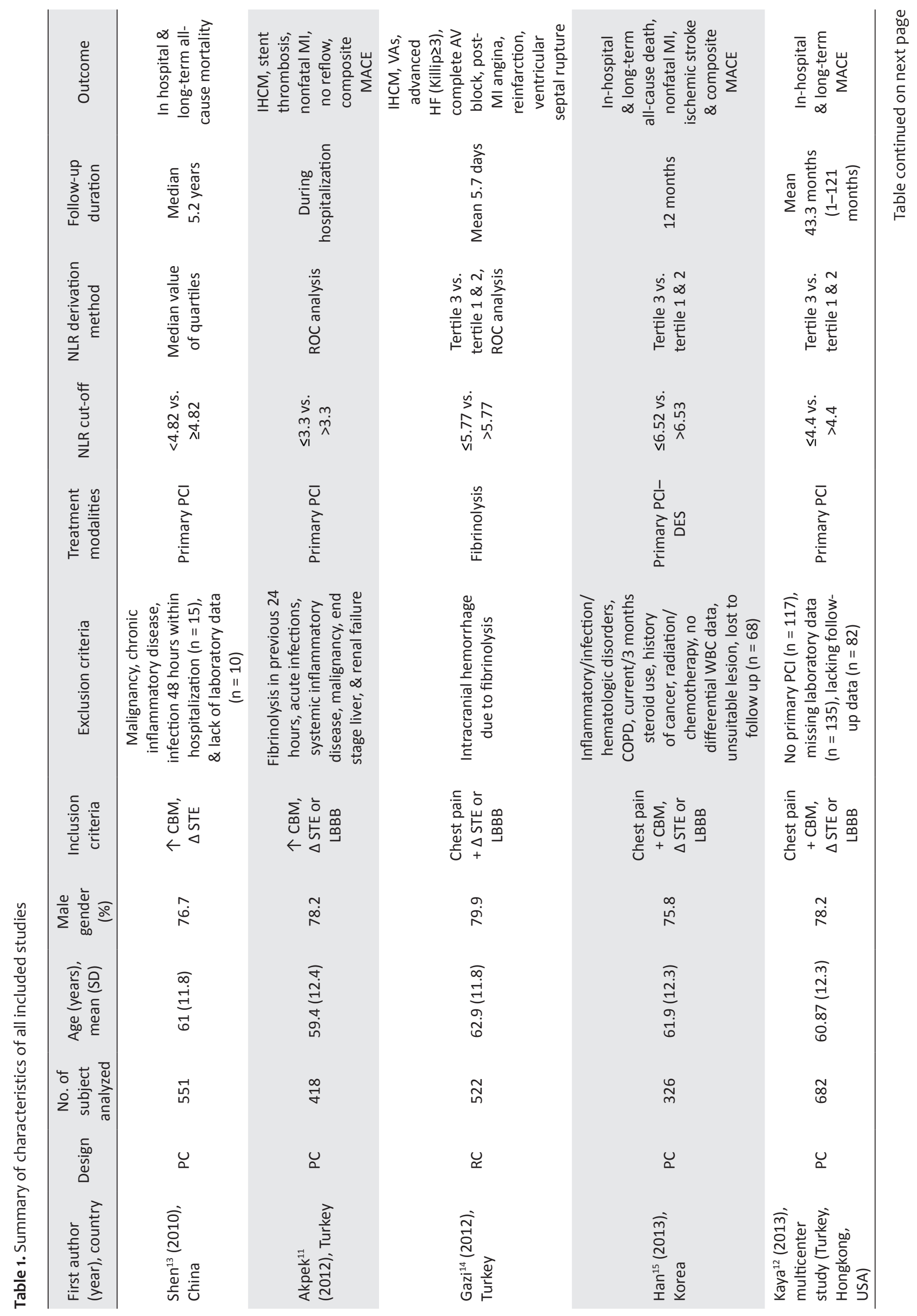




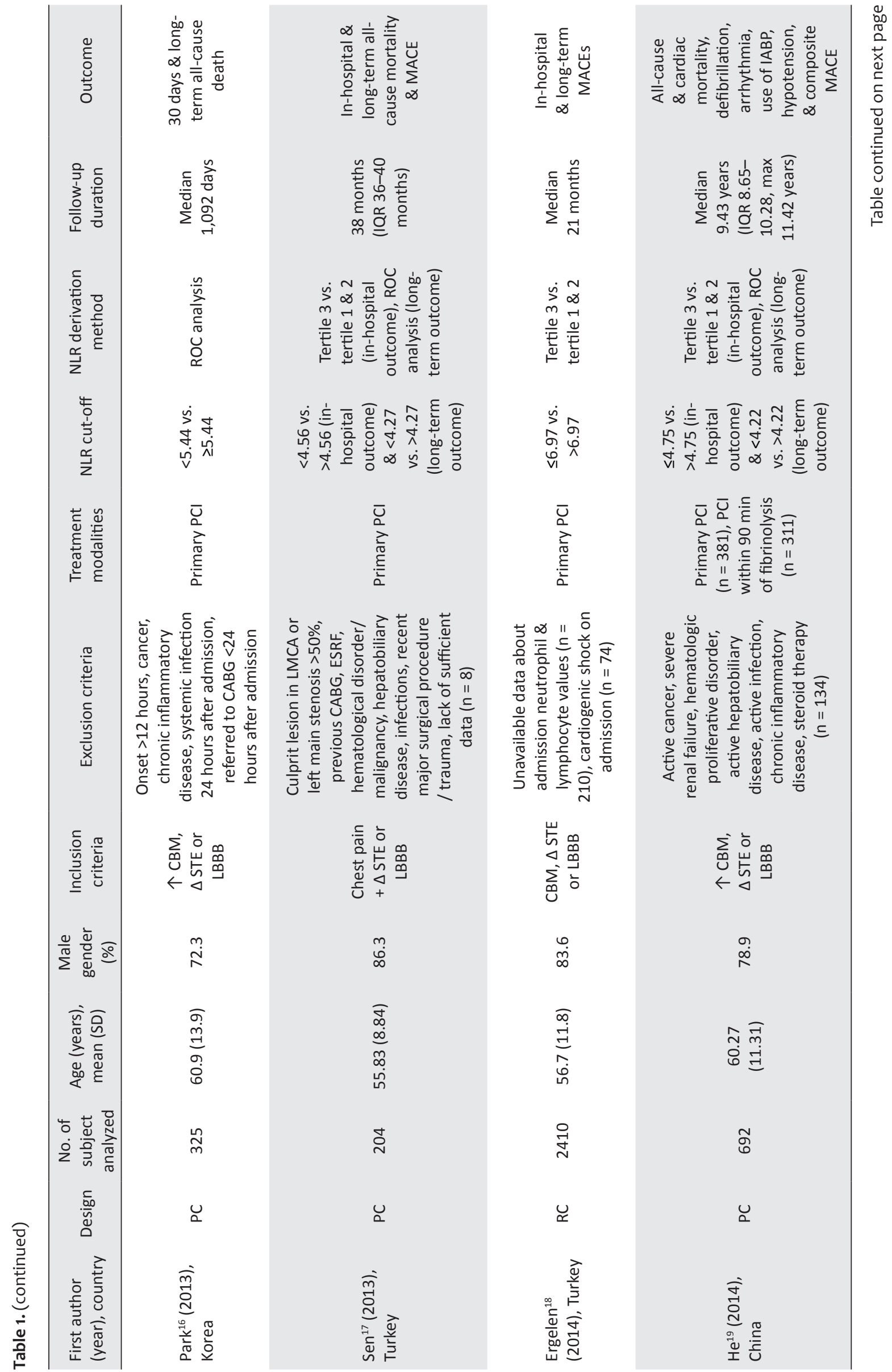




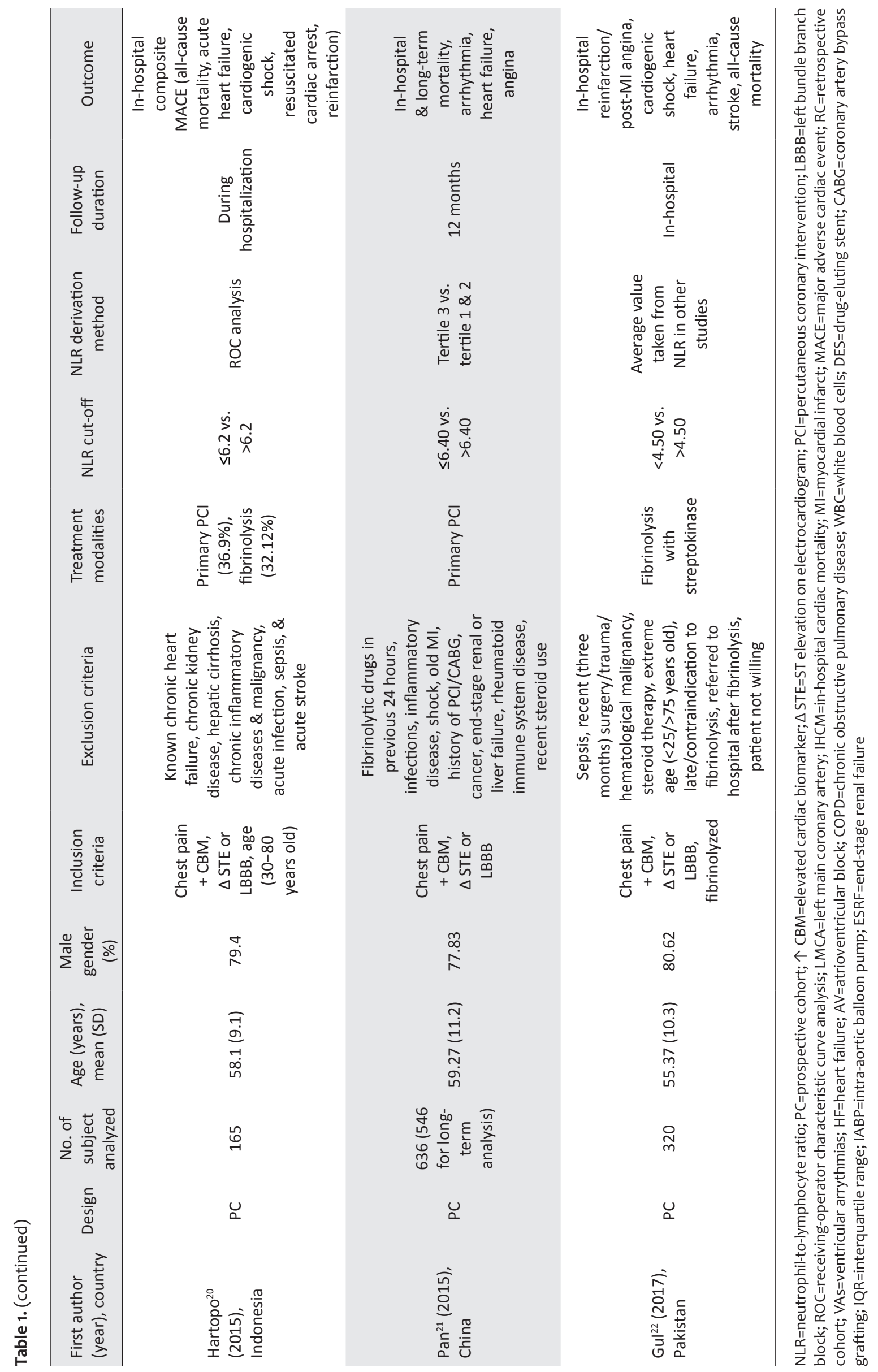


Table 2. Newcastle-Ottawa scale for quality assessment of studies
Figure 2. Funnel plot diagrams for bias assessment of studies. SE (log $[\mathrm{OR}])=$ standard error by log odds ratio

\begin{tabular}{|c|c|c|c|c|c|c|c|c|}
\hline \multirow[b]{2}{*}{ Study, year } & \multicolumn{4}{|c|}{ Selection } & \multirow[b]{2}{*}{$\begin{array}{l}\text { Comparability } \\
\text { of cohort } \\
\text { on the basis } \\
\text { of design or } \\
\text { analysis }\end{array}$} & \multicolumn{3}{|c|}{ Outcome } \\
\hline & 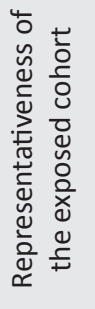 & 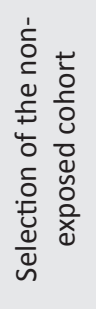 & 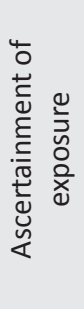 & 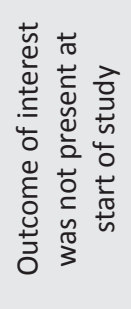 & & 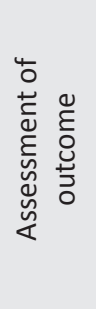 & 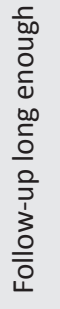 & $\begin{array}{l}0 \\
7 \\
3 \\
0 \\
0 \\
\frac{0}{4} \\
40 \\
\frac{0}{0} \\
\frac{0}{3} \\
\frac{0}{0} \\
\frac{0}{8}\end{array}$ \\
\hline Akpek, ${ }^{11} 2012$ & $*$ & $*$ & $*$ & $*$ & & $*$ & $*$ & $*$ \\
\hline Ergelen, ${ }^{18} 2014$ & $*$ & $*$ & $*$ & $*$ & & $*$ & $*$ & $*$ \\
\hline Gul, ${ }^{22} 2017$ & $*$ & $*$ & $*$ & $*$ & & $*$ & $*$ & $*$ \\
\hline Gazi, ${ }^{14} 2012$ & $*$ & $*$ & $*$ & $*$ & & $*$ & * & $*$ \\
\hline Han, ${ }^{15} 2013$ & $*$ & $*$ & $*$ & $*$ & & $*$ & $*$ & $*$ \\
\hline Hartopo, ${ }^{20} 2015$ & $*$ & $*$ & $*$ & $*$ & & $*$ & $*$ & $*$ \\
\hline $\mathrm{He},{ }^{19} 2014$ & $*$ & $*$ & $*$ & $*$ & & $*$ & $*$ & $*$ \\
\hline Kaya, ${ }^{12} 2013$ & $*$ & $*$ & * & $*$ & & $*$ & * & $*$ \\
\hline Pan, ${ }^{21} 2015$ & $*$ & $*$ & $*$ & $*$ & & $*$ & $*$ & $*$ \\
\hline Park, ${ }^{16} 2013$ & $*$ & $*$ & $*$ & $*$ & & $*$ & $*$ & $*$ \\
\hline Sen, ${ }^{17} 2013$ & $*$ & $*$ & $*$ & $*$ & & $*$ & $*$ & $*$ \\
\hline Shen, ${ }^{13} 2010$ & $*$ & $*$ & $*$ & $*$ & & $*$ & $*$ & $*$ \\
\hline
\end{tabular}

*Compatible with criterion listed below. All included studies were found to have good quality

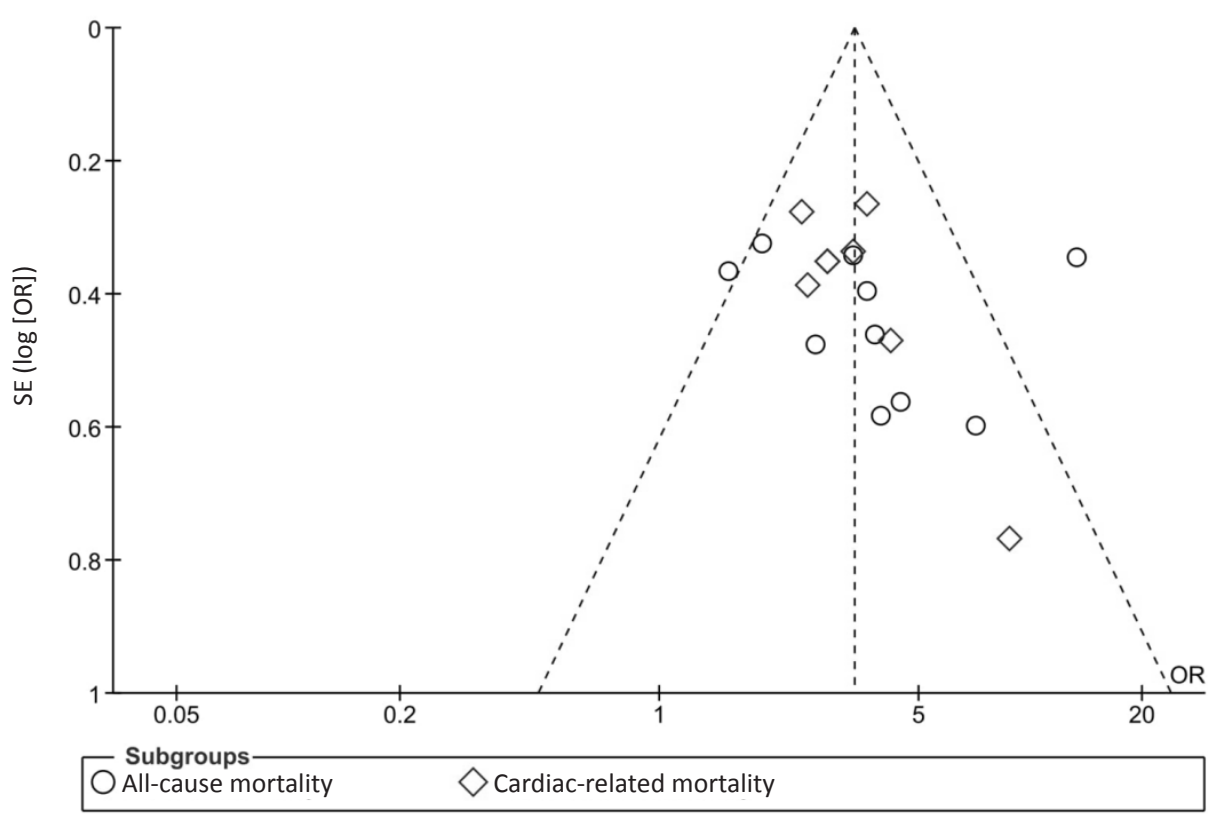

NLR are being widely used for diagnostic and prognostic purposes in various conditions, such as in solid tumors, ${ }^{26-28}$ autoimmune diseases, ${ }^{29}$ and infectious disease, ${ }^{30}$ due to its simplicity and availability. ${ }^{31}$ Its usage in cardiovascular disease are now being studied, such as in cerebrovascular disease, ${ }^{32}$ coronary artery disease, and acute coronary syndrome. ${ }^{33}$ Many studies have observed that elevated NLR level are related with cardiovascular complications in STEMI, such as lower ejection fraction, higher cardiac marker levels, and cardiac remodeling. ${ }^{34}$

Currently the most widely used prognostic score for predicting mortality in patients with STEMI is TIMI risk score. ${ }^{35}$ There is an agreement that an elevated NLR level is related with higher TIMI Score. ${ }^{36}$ Another predictors that have been studied are Killip class and 


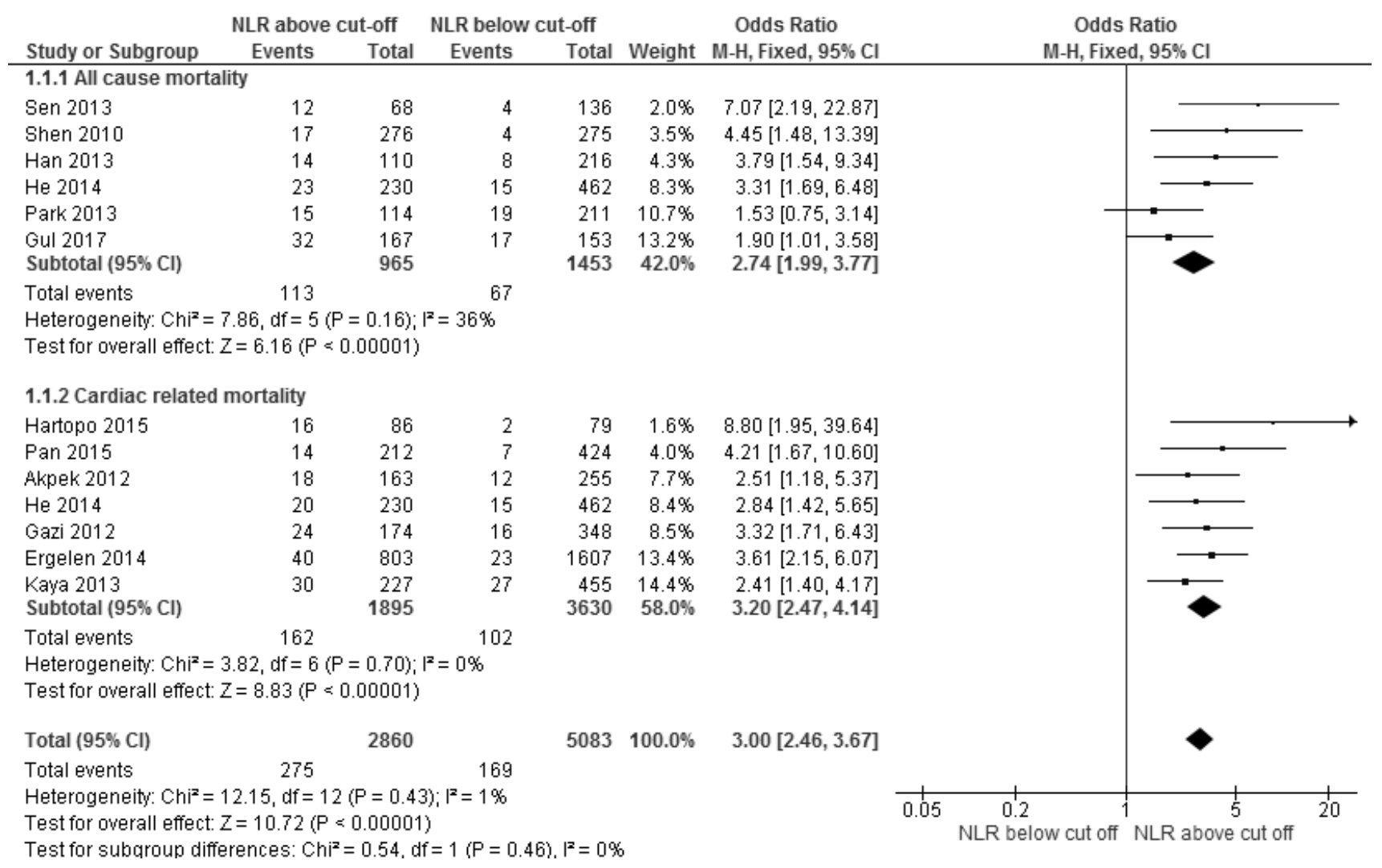

Figure 3. Forest plot diagram analysis of NLR to predict in-hospital mortality, either all-cause or cardiac-related mortality. $\mathrm{NLR}=$ neutrophil-to-lymphocyte ratio; $\mathrm{Cl}=$ confidence interval; $\mathrm{df}=$ degrees of freedom

glomerular filtration rate. ${ }^{37}$ We propose that NLR being used as a prognostic tool for STEMI patients, or incorporated with another risk factor into a scoring system. However the optimal cut-off is still needed to be determined.

This study found that NLR emerged as the potential new biomarker in STEMI since its potential to predict in-hospital mortality, especially cardiacrelated mortality. Thus, patient with high NLR value has a higher risk of death related to all-cause mortality and cardiac-related mortality during hospitalization. These findings suggest that NLR reflects the equilibrium between neutrophil and lymphocyte levels and indicates systemic inflammation. ${ }^{7}$ The increment of neutrophil level in blood demonstrates the signs of tissue injury and necrosis in STEMI, showing protein denaturation, which induces interleukin (IL)8, chemotaxin, IL-1, and tumor necrosis factor. Those immune reactions encourage neutrophil recruitment and increase vascular permeability to execute the function of phagocytosis. ${ }^{38}$ On the other hand, a proposed mechanism is that lymphopenia was a stress response secondary to increased body cortisol levels. ${ }^{39}$
This meta-analysis has certain limitations. First, all studies in this meta-analysis do not have single cut-off values for NLR. Each study analyzed and firmly established their specific cut-off values from the population samples. Moreover, the NLR cutoff values were set by using various methods. Out of 12 studies, 8 utilized tertiles or interquartiles range to determine the NLR cut-off values of the study population. By contrast, receiving-operator characteristic curve analyses were used to determine the NLR cut-off values by three out of 12 studies. As a result, the multiple cut-off values were observed in those analyses. Second, an elevated NLR predicted poor prognosis of in-hospital mortality among STEMI patients, but an exact single cut-off value of the NLR that carries worse prognosis cannot be determined. In the future, another prognostic study with a higher number of samples needs to be conducted. Lastly, this meta-analysis carried a high risk of publication bias due to asymmetry of the funnel plot diagrams. Furthermore, unpublished studies with "negative" results might have been missed. However, several factors may potentially influence the results of publication bias analysis by using the funnel plot, such 
as a small number of populations involved in those studies, which may affect asymmetrical findings in the funnel plot.

NLR was selected because it is inexpensive, practical, and widely available blood test, especially in limited health care facilities. Therefore, throughout this findings, we emphasized that the routine blood examination of NLR was compulsory in patients with STEMI, which could be done at any stage of the medical health service. Subsequently, NLR might be included in the risk assessment scoring in STEMI as an additional independent predictor for in-hospital or long-term MACE. From the clinicians' point of view, the prediction definitely would influence the subsequent treatments. Nevertheless, larger STEMI registries were required to fix single cut-off values of NLR determination. In conclusion, this meta-analysis demonstrated that elevated NLR predicted a higher risk of in-hospital mortality among STEMI patients.

\section{Conflict of Interest}

The authors affirm no conflict of interest in this study.

\section{Acknowledgment}

None.

\section{Funding Sources}

None.

\section{REFERENCES}

1. Sanchis-Gomar F, Perez-Quilis C, Leischik R, Lucia A. Epidemiology of coronary heart disease and acute coronary syndrome. Ann Transl Med. 2016;4(13):256.

2. Roth GA, Huffman MD, Moran AE, Feigin V, Mensah GA, Naghavi $\mathrm{M}$, et al. Global and regional patterns in cardiovascular mortality from 1990 to 2013. Circulation. 2015;132(17):1667-78.

3. Kusuma D. Cardiovascular diseases of multiple ethnic in Indonesia. In: Yuniadi Y, Hermanto DY, Raajoe AU. Textbook of cardiovascular volume 1. 1st ed. Jakarta: Sagung Seto; 2017. p. 3-17. Indonesian.

4. Hamm CW, Bassand JP, Agewall S, Bax J, Boersma E, Bueno $\mathrm{H}$, et al. ESC guidelines for the management of acute coronary syndromes in patients presenting without persistent STsegment elevation: The Task Force for the management of acute coronary syndromes (ACS) in patients presenting without persistent ST-segment elevation of the European Society of Cardiology (ESC). Eur Heart J. 2011;32(23):2999-3054.

5. Wilder J, Sabatine MS, Lilly LS. Acute coronary syndrome. In: Creager MA, Beckman JA, Losclazo J. Vascular medicine - a companion to Braunwald's heart disease. 2nd ed. Philadelphia: Elsevier Saunders; 2013. p. 162-91.

6. Shahawy S, Libby P. Atherosclerosis. In: Lilly LS. Pathophysiology of heart disease. 6th ed. Philadelphia: Lippincott William \& Wilkins; 2016. p. 112-32.

7. Horne BD, Anderson JL, John JM, Weaver A, Bair TL, Jensen $K R$, et al. Which white blood cell subtypes predict increased cardiovascular risk? J Am Coll Cardiol. 2005;45(10):1638-43.

8. Cho KH, Jeong MH, Ahmed K, Hachinohe D, Choi HS, Chang SY, et al. Value of early risk stratification using hemoglobin level and neutrophil-to-lymphocyte ratio in patients with ST-elevation myocardial infarction undergoing primary percutaneous coronary intervention. Am J Cardiol. 2011;107(6):849-56.

9. Núñez J, Núñez E, Bodí V, Sanchis J, Mainar L, Miñana G, et al. Low lymphocyte count in acute phase of ST-segment elevation myocardial infarction predicts long-term recurrent myocardial infarction. Coron Artery Dis. 2010;21(1):1-7.

10. Sawant AC, Adhikari P, Narra SR, Srivatsa SS, Mills PK, Srivatsa SS. Neutrophil to lymphocyte ratio predicts short- and long-term mortality following revascularization therapy for ST elevation myocardial infarction. Cardiol J. 2014;21(5):500-8.

11. Akpek M, Kaya MG, Lam YY, Sahin O, Elcik D, Celik T, et al. Relation of neutrophil/lymphocyte ratio to coronary flow to in-hospital major adverse cardiac events in patients with STelevated myocardial infarction undergoing primary coronary intervention. Am J Cardiol. 2012;110(5):621-7.

12. Kaya MG, Akpek M, Lam YY, Yarlioglues M, Celik T, Gunebakmaz $O$, et al. Prognostic value of neutrophil/lymphocyte ratio in patients with ST-elevated myocardial infarction undergoing primary coronary intervention: a prospective, multicenter study. Int J Cardiol. 2013;168(2):1154-9.

13. Shen XH, Chen Q, Shi Y, Li HW. Association of neutrophil/ lymphocyte ratio with long-term mortality after ST elevation myocardial infarction treated with primary percutaneous coronary intervention. Chin Med J (Engl). 2010;123(23):343843.

14. Gazi E, Bayram B, Gazi S, Temiz A, Kirilmaz B, Altun B, et al. Prognostic value of the neutrophil-lymphocyte ratio in patients with ST-elevated acute myocardial infarction. Clin Appl Thromb Hemost. 2012;21(2):155-9.

15. Han YC, Yang TH, Kim DI, Jin HY, Chung SR, Seo JS, et al. Neutrophil to lymphocyte ratio predicts long-term clinical outcomes in patients with ST-segment elevation myocardial infarction undergoing primary percutaneous coronary intervention. Korean Circ J. 2013;43(2):93-9.

16. Park JJ, Jang HJ, Oh IY, Yoon CH, Suh JW, Cho YS, et al. Prognostic value of neutrophil to lymphocyte ratio in patients presenting with ST-elevation myocardial infarction undergoing primary percutaneous coronary intervention. Am J Cardiol. 2013;111(5):636-42.

17. Sen N, Afsar B, Ozcan F, Buyukkaya E, Isleyen A, Akcay AB, et al. The neutrophil to lymphocyte ratio was associated with impaired myocardial perfusion and long term adverse outcome in patients with ST-elevated myocardial infarction undergoing primary coronary intervention. Atherosclerosis. 2013;228(1):203-10.

18. Ergelen M, Uyarel H, Altay S, Kul S, Ayhan E, Isik T, et al. Predictive value of elevated neutrophil to lymphocyte ratio in patients undergoing primary angioplasty for ST-segment elevation myocardial infarction. Clin Appl Thromb Hemost. 2014;20(4):427-32.

19. He J, Li J, Wang Y, Hao P, Hua Q. Neutrophil-to-lymphocyte ratio (NLR) predicts mortality and adverse-outcomes after STsegment elevation myocardial infarction in Chinese people. Int J Clin Exp Pathol. 2014;7(7):4045-56.

20. Hartopo AB, Puspitawati I, Setianto BY. On-admission high neutrophil to lymphocyte ratio as predictor of in-hospital adverse cardiac event in st-elevation myocardial infarction. Acta Med Indones. 2015;47(1):3-10.

21. Pan W, Zhao D, Zhang C, Li W, Yu J, Wang S, et al. Application of neutrophil/lymphocyte ratio in predicting coronary blood flow and mortality in patients with ST-elevation myocardial infarction undergoing percutaneous coronary intervention. J Cardiol. 2015;66(1):9-14.

22. Gul U, kayani AM, Munir R, Hussain S. Neutrophil lymphocyte ratio: a prognostic marker in acute st elevation myocardial infarction. J Coll Physicians Surg Pak. 2017;27(1):4-7.

23. Dharma S, Andriantoro H, Purnawan I, Dakota I, Basalamah F, Hartono B, et al. Characteristics, treatment and in-hospital 
outcomes of patients with STEMI in a metropolitan area of a developing country: an initial report of the extended Jakarta Acute Coronary Syndrome registry. BMJ Open. 2016;6(8):e012193.

24. Miyachi H, Takagi A, Miyauchi K, Yamasaki M, Tanaka H, Yoshikawa $M$, et al. Current characteristics and management of ST elevation and non-ST elevation myocardial infarction in the Tokyo metropolitan area: from the Tokyo CCU network registered cohort. Heart Vessels. 2016;31(11):1740-51.

25. Fox KA, Eagle KA, Gore JM, Steg PG, Anderson FA, GRACE and GRACE2 Investigators. The Global Registry of Acute Coronary Events, 1999 to 2009 - GRACE. Heart. 2010;96(14):1095-101.

26. Demirer Z, Uslu AU. Predictive value of neutrophil-lymphocyte ratio in non-muscle-invasive bladder cancer. Urol Oncol. 2016;34(1):1-2.

27. Cho Y, Kim WH, Yoon HI, Lee CG, Keum KC, Lee IJ. The prognostic significance of neutrophil-to-lymphocyte ratio in head and neck cancer patients treated with radiotherapy. J Clin Med. 2018;7(12):512.

28. Deng $M$, Ma X, Liang C, Zhu C, Wang M. Are pretreatment neutrophil-lymphocyte ratio and platelet-lymphocyte ratio useful in predicting the outcomes of patients with small-cell lung cancer? Oncotarget. 2017;8(23):37200-7.

29. Li L, Xia Y, Chen C, Cheng P, Peng C. Neutrophil-lymphocyte ratio in systemic lupus erythematosus disease: a retrospective study. Int J Clin Exp Med. 2015;8(7):11026-31.

30. Martins EC, da Fe Silveira L, Viegas K, Beck AD, Júnior GF, Cremonese RV, et al. Neutrophil-lymphocyte ratio in the early diagnosis of sepsis in an intensive care unit: a case control study. Rev Bras Ter Intensiva. 2019;31(1):63-70.

31. Uslu AU, Küçük $A$, Şahin A, Ugan $Y$, Yilmaz R, Güngör $T$, et al. Two new inflammatory markers associated with Disease Activity Score-28 in patients with rheumatoid arthritis: neutrophillymphocyte ratio and platelet-lymphocyte ratio. Int J Rheum Dis. 2015;18(7):731-5.
32. Zhang J, Ren Q, Song Y, He M, Zeng Y, Liu Z, et al Prognostic role of neutrophil-lymphocyte ratio in patients with acute ischemic stroke. Medicine (Baltimore). 2017;96(45);e8624.

33. Angkananard T, Anothaisintawee T, Mc Evoy M, Attia J, Thakkinistian A. Neutrophil lymphocyte ratio and cardiovascular disease risk: a systematic review and meta-analysis. Biomed Res Int. 2018;2018:2703518.

34. Börekçi A, Gür M, Türkoğlu C, Baykan AO, Şeker T, Şahin DY, et al. Neutrophil to lymphocyte ratio predicts left ventricular remodeling in patients with ST elevation myocardial infarction after primary percutaneous coronary intervention. Korean Circ J. 2016;46(1):15-22.

35. Morrow DA, Antman EM, Charlesworth A, Cairns R, Murphy SA, de Lemos JA, et al. TIMI risk score for ST-elevation myocardial infarction: a conventient, bedside, clinical score for risk assessment at presentation: an intravenous nPA for treatment of infarcting myocardium early II trial substudy. Circulation. 2000;102(17):2031-7.

36. Acet H, Ertaş F, Bilik MZ, Akıl MA, Özyurtlu F, Aydın M, et al. The relationship between neutrophil to lymphocyte ratio, platelet to lymphocyte ratio and thrombolysis in myocardial infarction risk score in patients with ST elevation acute myocardial infarction before primary coronary intervention. Postepy Kardiol Interwecyjnej. 2015;11(2):126-35.

37. Ginanjar E, Yamin M, Wijaya IP, Harimurti K. Predictors of 30-day mortaliy in ST-elevation myocardial infarction (STEMI) patients. Acta Med Indones. 2019;51(3):238-43.

38. Meeuwsen JA, Wesseling M, Hoefer IE, de Jager SC. Prognostic value of circulating inflammatory cells in patients with stable and acute coronary artery disease. Front Cardiovasc Med. 2017;4:44.

39. Widmer A, Linka AZ, Attenhofer Jost CH, Buergi B, BrunnerLa Rocca HP, Salomon F, et al. Mechanical complications after myocardial infarction reliably predicted using $\mathrm{C}$-reactive protein levels and lymphocytopenia. Cardiology. 2003;99(1):25-31. 